\title{
The co-evolution of organizational and network structure: The role of multilevel mixing and closure mechanisms
}

\begin{abstract}
We present a dynamic multilevel framework for analyzing the mutual dependence of change in interorganizational networks and internal organizational structure. Change occurring at the former (interorganizational) level involves decisions to change the portfolio of network ties to external partners. Change occurring in the latter (intraorganizational) level involves decisions to change the portfolio of internal activities. We estimate a recently derived class of stochastic actor-oriented models (SAOMs) that we adopt and adapt to specify how decisions to change internal portfolios of activities and external portfolios of partners are connected by theoretically derived multilevel mechanisms that link organizational and network structures. We show that statistical models for multilevel networks reproduce with high fidelity the structural regularities observed in the distribution of: (i) activities within organizations; (ii) network ties between organizations, and (iii) knowledge available in the organizational field. We discuss the implications of the study for theory development, and for empirical research on interorganizational and other kinds of multilevel networks.
\end{abstract}

Keywords: Generalized linear models, Health care organizations; Interorganizational networks, Multilevel networks, Network evolution, Organizational change, Organizational fields, Social mechanisms, Stochastic actor-oriented models. 


\section{Introduction}

One of the most enduring insights of organization theory is the recognition of formal organizations as hierarchical multilevel social systems (Simon, 1962). Almost by definition, therefore, building and testing network theories of organizations involve the development of models for multilevel networks (Brass et al., 2004; Moliterno and Mahony, 2011; Zappa and Lomi, 2015). This point is particularly salient for studies of interorganizational fields (Powell et al., 2005) where "each node in a network at a given level of analysis is itself a network at a lower level of analysis" (Moliterno and Mahony, 2011, p.444). In this paper we develop further this fundamental insight and link it to recently derived models for the analysis of multilevel networks (Snijders et al., 2013).

Understanding the relation between change in internal organizational structure and change in interorganizational networks involves specifying the multilevel mechanisms linking two sub-systems of organizational decisions. The first concerns decisions about the creation and maintenance of portfolios of internal resources (activities) that organizations accumulate over time through investments in production capacity (Cohen and Levinthal, 1994). The second concerns decisions about the composition of portfolios of partners that organizations construct over time through investments in networks of relations (Powell et al., 1996). How are these sets of decisions related, and how do they affect one another over time? Addressing these questions is central to our understanding of how organizations construct the social space that they inhabit by reconfiguring, simultaneously, their internal structures and their networks of external dependence relations (Hollway et al., 2017). 
Building on recently proposed class of stochastic actor-oriented models (SAOMs) for multiple networks (Snijders et al., 2013), we present statistical models that assist in specifying the dynamic multilevel social mechanisms shaping the co-evolution of interorganizational networks and internal organizational structures. The mechanisms at the heart of our models are "dynamic" because they generate observable structural change. They are "multilevel" because they involve change simultaneously unfolding at the intra- and interorganizational level. These mechanisms are "social" because they are defined in terms of relations connecting nodes embedded in networks of interorganizational relations. Finally, the explanatory processes at the core of our models are "mechanisms" in the sense of Schelling (1998, pp. 32-33), because they represent hypotheses about how observed macro-structures may be explained in terms of patterns of local relations between social agents - organizations in our case. We focus on the analysis of two main classes of multilevel mechanisms connecting internal and external processes of organizational and network change.

Building on the original model proposed by Snijders et al. (2013), the first class of multilevel mechanisms connect changes in the number of internal organizational activities to changes in the number of network partners. Extending established single-level network concepts (Newman, 2003), we call this family of mechanisms multilevel mixing. The second class of multilevel mechanisms connect changes in the composition of internal organizational activities to changes in the composition of external networks of partners. Specifying the intuition developed by Easley and Kleinberg (2010) in their discussion of multi-mode networks, we call this class of mechanisms multilevel closure. We estimate SAOMs that specify how multilevel 
mixing and closure mechanisms concatenate to shape the joint dynamics of organizational structures and interorganizational networks. Following best practice in dynamic network modeling, we adopt computational methods to validate the fitted model and assess its closeness to the observed multilevel data (Hunter et al., 2008; Snijders and Steglich, 2015).

To establish the empirical value of our methodological proposal we examine longitudinal data we have collected on interorganizational networks and internal activities of health care organizations operating in a small regional community (Stadtfeld et al., 2016). We selected the field of health care because it illustrates particularly well how internal organizational design decisions and external partner selection decisions are related (Gittell and Weiss, 2004; Shortell et al., 1993). We reconstruct interorganizational networks in terms of collaborative patient referral relations connecting partner hospitals (Mascia et al., 2017). We define internal organizational structures in terms of dynamic bipartite (more precisely, 2-mode) networks affiliating individual organizations to their internal clinical activities (Conaldi et al., 2012).

The multilevel network structure that we examine in the empirical part of the paper is obviously specific to our focus on interorganizational relations where organizations are seen as network "nodes" with an internal structure that may itself be represented as a (bipartite) network. It behooves us to clarify at the outset that our example does not limit in any way the levels of organizational analysis that are amenable to be examined through the multilevel network model we propose. Teams and individuals (at a more "micro" level), and markets and organizations (at a 
more "macro" level) are examples of multilevel contexts that would lend themselves equally well to be examined through the multilevel network lens that we propose in this paper. In general, once the source of action (the "actor") has been identified unambiguously, the model is indifferent to the specific choice of level of analysis.

In the specific context of our study, the source of action is the individual hospital at the crossroad of two distinct, yet interdependent sets of decisions. The first is the decision to rely on a partner hospital for help with the resolution to a clinical problem represented by a patient. The second is the decision to modify its formal structure by adding or removing formal sub-units responsible for specific clinical activities. These two sets of decisions are not independent because the capacity of an hospital to provide efficient and high quality care to patients depends on how these decisions are coordinated.

Multilevel mixing mechanisms connect the number of external partners (i.e., the outdegree in the interorganizational network), to the number of internal activities that hospitals hold in their portfolios (i.e., the outdegree in the affiliation network). Multilevel closure mechanisms may derive from the preferential tendency of connected hospitals to become more similar by emulating or incorporating the activities of partners, and/or from the tendency of similar hospitals (i.e., hospitals containing similar internal activities) to become partners. Following Easley and Kleinberg (2010), we call the former closure mechanism "multilevel membership closure" and the the latter "multilevel focal closure." Membership closure involves processes of social influence whereby connection triggers emulation and diffusion. Focal closure involves processes of social selection whereby similar organizations are more likely to 
become partners (Steglich et al., 2010). The model we present in this paper allows us to discriminate between these two alternative sub-processes that may be underlying the tendency of interdependent organizations to be (or become) more similar, i.e., the tendency toward mimetic isomorphism (DiMaggio and Powell, 1983). ${ }^{1}$

We show that a model specified in terms of these various multilevel micromechanisms reproduces with a very high level of fidelity observed macro-regularities in the: (i) distribution of network ties between organizations; (ii) composition of activities within organizations, and (iii) diffusion of knowledge within the organizational community.

\section{Motivation and Background}

\subsection{Connected portfolios}

According to Padgett et al. (2003, p.843) "Firms and the core competencies that define firms are developed and maintained through learning by doing and other learning processes that are triggered by the exchange among firms." In this perspective, organizations are defined by contingent combinations of proprietary internal resources that are controlled directly, and extramural resources accessible indirectly through relations with network partners. Internal resources provide the basis of experiential learning (Cohen and Levinthal, 1994). External resources involve a process of partner selection - i.e, a process of sampling, and learning from, the experience of others (Beckman and Haunschild, 2002; Denrell, 2003; Greve, 2005). Portfolios of

\footnotetext{
${ }^{1}$ In a different context, Lomi and Stadtfeld (2014) call membership closure "association-based closure" and focal closure "affiliation-based closure."
} 
internal and external resources are connected and mutually dependent. Change in the composition of one may induce change in the composition of the other. This happens because change in internal resources provides the basis for new external dependencies (Pfeffer and Salancik, 2003). At the same time, change in networks of external partners provides new opportunities for learning, emulation and differentiation that may be achieved through change in internal activities (Stadtfeld et al., 2016). In more general terms, a distinctive feature of multilevel network systems is their ability to transmit change across connected levels - regardless of the specific level at which change action is initiated.

Observed configurations of internal organizational activities are the cumulative outcome of decisions to invest in productive capacity. Observed interorganizational configurations are the cumulative outcome of decisions to invest in relations with external partners. How are these two systems of decisions connected? Addressing this question requires explicit identification of multilevel dependence mechanisms linking change in internal organizational structure to change in external networks with partners.

Existing lines of research on organizational change (Barnett and Carroll, 1995) and network evolution (Ahuja et al., 2012) have remained surprisingly - but understandably - disjoint. We say surprisingly disjoint because the theoretical connection between internal and external knowledge and organizational resource accumulation processes is generally acknowledged (Cohen and Levinthal, 1994; Gibbons and Henderson, 2012; Gulati, 1999; Lorenzoni and Lipparini, 1999). We say understandably disjoint because of the lack of a general analytical framework to represent the multi- 
level mechanisms linking organizational and network change (DiMaggio, 1986; Hitt et al., 2007). Influential statements of multilevel perspectives on organizations and social networks clearly recognize this problem (Borgatti and Foster, 2003; Brass et al., 2004; Contractor et al., 2006), but offer little guidance about the specific mechanisms that connect these multiple levels. In this paper, we begin to fill this gap by proposing one such framework, and testing its empirical value on a realistically complex empirical case study (Stadtfeld et al., 2016).

As necessary background to our methodological proposal, it may be useful to recall the fundamental distinction between multilevel network analysis and analysis of multilevel networks recently delineated by Snijders (2016). This distinction is at the center of the current debate on the analysis of social and other kinds of networks (Lomi et al., 2016; Wang et al., 2016). Multilevel network analysis involves the combined analysis of social relations within multiple independent groups (Snijders, 2016) based on models with random coefficients. A recent example of applied multilevel network analysis is the study by Boda (2018) on social influence and ethnic identity in multiple classes of high school students. The analysis of multilevel networks involves the analysis of networks containing different kinds of nodes linked by different kinds of relations (Lomi et al., 2016; Wang et al., 2013; Snijders, 2016). A recent example of analysis of multilevel networks is the study by Lomi et al. (2017) on relations among managers and, simultaneously, between managers and words they use to describe their company.

In the analysis we present in this paper, we adopt a recently developed class of stochastic actor-oriented models for the analysis of multilevel networks. Interorga- 
nizational relations offer a particularly appropriate setting for illustrating the value of dynamic multilevel network concepts and models. This is the case because organizations are network nodes with an internal structure that is differentiated and changeable (DiMaggio, 1986). When organizational structure changes, the most fundamental attachment mechanisms such as, for example, homophily and reciprocity become problematic as the working of these mechanisms depends delicately on assumptions about the stability of organizational identities (Padgett and Powell, 2012). Under conditions of organizational transformation there is no reason to expect that attachment mechanisms typically operating in equilibrium will continue to operate among partners. Change in networks of partners that is likely to ensue, disrupts patterns of resource dependence that organizations typically manage by rearranging their internal structure - thus creating the basis for new exchange and dependence relations (Pfeffer and Salancik, 2003). In studies of interorganizational networks, this situation provides an almost ideal illustration of this kind of multilevel change discussed by Kozlowski and Klein (2000). The model that we propose and illustrate incorporates these coupled multilevel mechanisms of change in the more general context of stochastic actor-oriented models for dynamic networks.

\subsection{Multilevel network mechanisms}

Following recent research in the statistical analysis of social networks (Robins et al., 2009; Snijders, 2016), we focus on multilevel mechanisms that regulate connectivity and clustering - the major structural features of interorganizational networks (Baum et al., 2003). The former class of mechanisms control dependencies linking the num- 
ber of internal activities and the number of external partners - or, in other words, the tendency of organizations with different niche widths to establish various types of community relations (Barnett and Carroll, 1987). We call these mechanisms multilevel mixing because they involve degree correlations across levels of observation (Newman, 2003). The latter class of mechanisms assist with modeling extra-dyadic dependencies regulating multilevel closure - or the tendency of interdependent organizations to be similar (DiMaggio and Powell, 1983). We call these multilevel closure mechanisms because their effect is to close open configurations of internal activities and external network ties (multilevel 2-paths, in network parlance). We now discuss these various mechanisms more precisely.

\subsection{Multilevel mixing}

A distinctive feature of social networks is the general tendency of nodes with similar degrees to associate, typically referred to as " assortative mixing"' (Newman, 2003). In the context of interorganizational networks, the number of partners depends, at least in part, on the composition of the portfolio of internal activities managed by the organizations involved. This is the case because partner selection depends on a comparative assessment of compatibilities and complementarities in the internal portfolios of resources that partners control (Mitsuhashi and Greve, 2009). As the composition of internal activities changes, it is likely that the composition and number of partner will also change. This may happen, for example, because change in the composition of internal activities implies change in patterns of niche overlap within organizational communities and fields (Podolny et al., 1996). In turn, change 
in patterns of niche overlap will determine new conditions for the emergence of competitive and collaborative relations among organizations within the field (Lomi and Pallotti, 2012).

In what direction causality flows (from change in composition of internal activities to change in composition of external partners, or vice versa) is an important issue that we address in the empirical part of the study. Similarly important is establishing the valence of the degree correlation that multilevel mixing mechanisms tend to induce. If a larger number of internal activities leads to a larger number of partners, the multilevel network associating internal and network structure is assortative. If the contrary holds, the multilevel network is disassortative. Identifying the specific shape assumed by multilevel mixing effects allows us to clarify the interdependence between internal portfolios of activities end external portfolios of partners. Figure 1 summarizes this argument.

- Figure 1 about here -

In panel (a) of Figure 1, a narrowly focused organization (i.e., a specialist organization holding only one activity in its internal portfolio) entertains exchange relations with many partners. This situation illustrates disassortative multilevel mixing, or (multilevel) disassortativity - a negative association between degrees of connected nodes across multiple network levels. In panel (b), a broadly focused organization (i.e., a generalist organization holding a broad portfolio of internal activities) relies on many external partners. This situation illustrates assortative multilevel mixing, or (multilevel) assortativity - a positive association between degrees of connected nodes across multiple network levels. In Figure 1 the direction of interorganizational 
relations is ignored in the interest of simplicity and clarity. However, the analogous configurations for directed relations can be derived by representing interorganizational relations as incoming or outgoing ties. As we will see in the empirical part of the paper, the mechanism-oriented approach we propose affords identification of the direction of causality underlying observationally equivalent configurations of ties produced by different multilevel mixing mechanisms.

\subsection{Multilevel closure}

The second distinctive structural feature of social networks is clustering - induced by the preferential tendency of pairs of nodes to be connected if they are both connected to one or more common third parties (Watts and Strogatz, 1998). An extensive organizational literature is available that demonstrates the tendency of interorganizational networks to cluster (Baum et al., 2003; Kogut and Walker, 2001) as a consequence of various path-shortening (or "closure") strategies (Lomi and Pattison, 2006; Lomi and Pallotti, 2013). Studies of how tendencies toward closure in interorganizational networks are affected by processes of internal organizational change, however, are far less common and have started to appear only very recently (Stadtfeld et al., 2016). This is striking in the light of the fact that one of the main theoretical propositions inspiring studies of interorganizational networks posits a multilevel relation linking external dependence relations and internal organizational structure. As DiMaggio and Powell (1983, p. 154) predicted: "The greater the dependence of an organization on another organization, the more similar it will become to that organization in structure, climate and behavioral orientation" (em- 
phasis added). This prediction can only be tested by linking external dependence relations to internal organizational structure, i.e., by developing a multilevel framework.

The empirical propensity of similar organizations to be connected that this prediction implies, may be generated by two very different multilevel closure mechanisms that tend to produce observationally equivalent outcomes. Adopting the terminology established by Easley and Kleinberg (2010) we may call the first mechanism focal closure - the preferential tendency of similar organizations (organizations sharing the same foci of activity) to establish interorganizational ties to manage their interdependence. The second multilevel mechanism is membership closure - the tendency of connected organizations (organizations members in the same dyad) to become more similar by incorporating the same internal activities held by partners (Easley and Kleinberg, 2010, pp. 95 -97). Focal and membership closure implicate very different multi-level mechanisms: The former involves social selection, the latter social influence (Steglich et al., 2010). Yet, they produce the same (cross sectional) outcome: similar organizations connected by network ties. Disentangling focal and membership closure amounts to establishing the relative weight of generalized processes of influence and selection on the co-evolutionary dynamics of interorganizational networks and organizational structures. Identifying which closure mechanism is effectively operating is essential to the correct causal understanding of the institutional process responsible for mimetic isomorphism in interorganizational fields.

- Figure 2 about here - 
Figure 2 illustrates this point. In panel 1, connected organizations (time T1) become more likely to share an activity (time T2), i.e., become more similar (multilevel membership closure). In panel 2 , organizations sharing an activity (i.e., similar organizations, time T1) are more likely to become connected (multilevel focal closure, time T2). As in Figure 1, the direction of interorganizational relations is ignored for simplicity, but without loss of generality.

\section{Stochastic actor-oriented models for multilevel}

\section{networks}

\subsection{Models for multilevel networks}

Models for multilevel networks involve explicit connections across levels, and hence across different classes of actors, or network nodes. According to Lomi et al. (2016, p. 266), models for multilevel networks include: "Distinct types of nodes defined at different multiple levels (e.g., individuals and groups) with ties possible between all nodes, both within and across levels (e.g., distinct types of dyadic ties at the individual - and the group-level, as well as individual-group affiliations)." Models for multilevel networks that have proven useful in empirical organizational research include multilevel exponential random graph models (Zappa and Lomi, 2015; Wang et al., 2013) and recent extensions of multiple membership multiple classification models (Tranmer et al., 2016, 2014). These models are typically defined for crosssectional data, and hence leave change mechanisms unspecified (Block et al., 2018). Also, in available models for multilevel networks individual agency plays only an 
implicit role as preferences of individual actors are left implicit.

Like other available models for multilevel networks, stochastic actor-oriented models (SAOMs) include a bipartite component (a meso-level network) that connects different units across different levels. Unlike available models SAOMs for multilevel networks are models for change - where change is explicitly represented as a consequence of individual preferences defined over local configurations of network ties. We discuss this next.

\subsection{Notation and model formulation}

Stochastic actor-oriented models are continuous-time models for network panel data (Snijders et al., 2010). A recent extension allows joint analysis of one-mode and twomode networks so that dependence mechanisms within and across networks can be specified rigorously (Snijders et al., 2013). In the case we examine in the empirical part of the paper, the one-mode network defines the system of collaborative relations among organizations. The two-mode network defines the internal organizational structure in terms of composition of portfolios of activities.

The model may be summarized as follows. Let $\mathcal{N}=\{1, \cdots, n\}$ be the set of nodes, and $\mathcal{A}=\{1, \cdots, a\}$ be the set of activities. The one-mode network is represented by an $n \times n$ adjacency matrix $X$ with cell $X_{i j}, i, j \in \mathcal{N}$, taking value 1 if there is a tie from $i$ to $j$ and 0 otherwise. The two-mode network is represented as an $n \times a$ adjacency matrix $Y$ with cell $Y_{i j}, i \in \mathcal{N}, j \in \mathcal{A}$, being 1 if hospital $i$ maintains clinical activity $j \in \mathcal{A}$ and 0 otherwise. In a panel design, the one-mode and the two-mode networks are observed at $M$ time points $t_{1}, \cdots, t_{M}$. We denote 
by $x(t)$ and $y(t)$ the observations at time $t$ of the one-mode and two-mode networks, respectively.

The model assumes that the observed networks are the outcome of a sequence of unobserved opportunities for tie changes taking place between two consecutive observations of the networks. Those opportunities are usually referred to as microsteps (Steglich et al., 2010). At each micro-step, an actor gets the opportunity to revise one of its outgoing ties, either in the one-mode or in the two-mode network, and decides whether to make a change (delete a tie if present and create a tie if absent) or do nothing. Formally, the model assumes that the observed networks are the outcome of a continuous-time Markov process, where at randomly occurring time points one node $i$ faces an opportunity to change either one tie in $X$ or one tie in $Y$. The waiting times for an opportunity of a tie change in the one-mode network $X$ or in the two-mode network $Y$ are described by exponential distributions with rate parameters $\lambda^{X}(x, y)$ and $\lambda^{Y}(x, y)$, respectively. The transition matrix of the process describes the probability of each possible change, conditional on the node that has the opportunity to make the change. These probabilities are defined by a multinomial logit model. Given an opportunity for change in the one-mode network, the probability that node $i$ changes the tie $x_{i j}$ into $1-x_{i j}$ is:

$$
P\left[i \text { changes } x_{i j} \mid Y(t)=y, X(t)=x\right]=\frac{\exp \left(f_{i}\left(x^{i j}, y\right)\right)}{\sum_{h \in \mathcal{N}} \exp \left(f_{i}\left(x^{i h}, y\right)\right)}
$$

where $x^{i j}$ denotes the network where the change is made in tie $x_{i j}$.

Given an opportunity for change in the two-mode network, the probability that node $i$ changes the tie $y_{i j}$ into $1-y_{i j}$ is: 


$$
P\left[i \text { changes } y_{i j} \mid Y(t)=y, X(t)=x\right]=\frac{\exp \left(g_{i}\left(x, y^{i j}\right)\right)}{\sum_{h \in \mathcal{A}} \exp \left(g_{i}\left(x, y^{i h}\right)\right)}
$$

where $y^{i j}$ denotes the network where the change takes place in tie $y_{i j}$.

The functions $f_{i}\left(x^{i j}, y\right)=\sum_{k} \beta_{k} s_{k}\left(x^{i j}, y\right)$ and $g_{i}\left(x, y^{i j}\right)=\sum_{k} \gamma_{k} s_{k}\left(x, y^{i j}\right)$ are referred to as "evaluation" functions. They are defined as linear combinations of statistics $s_{k}\left(x^{i j}, y\right)$ and $s_{k}\left(x, y^{i j}\right)$, and parameters $\beta_{k}$ and $\gamma_{k}$. The statistics $s_{k}\left(x^{i j}, y\right)$ and $s_{k}\left(x, y^{i j}\right)$ are counts of network configurations that embody specific hypothesis on the micro-mechanisms driving the network changes. The statistics $s_{k}$ represent mechanisms of general theoretical relevance, or contextual empirical importance. Examples of statistics in the one-mode network are the number of ties and the number of reciprocal dyads (i.e., pairs of nodes $(i, j)$ with both the ties from $i$ to $j$ and from $j$ to $i$ present) in which a node $i$ is embedded. Those statistics refer to the baseline tendency of nodes to establish ties and to the tendency of nodes to reciprocate existing ties. Examples of statistics in the two-mode network are the number of ties and the number of four-cycles (i.e., combinations of four ties connecting nodes $i$ and $j$ with both affiliations $a_{1}$ and $a_{2}$ ) in which a node $i$ is embedded. Those statistics describe the baseline tendency of nodes to connect to affiliations and the preferential tendency of nodes sharing an affiliation to get more affiliations in common. Examples of statistics involving one-mode and two-mode network ties are discussed in detail in the next section. An overview of the most common statistics is provided in Table 1 and discussed in Section 4.3. 


\subsection{Model interpretation}

The parameters $\beta_{k}$ and $\gamma_{k}$ describe whether the corresponding micro-mechanisms are relevant to explain the evolution of the networks over time. A positive (negative) value of a parameter indicates that ties leading to an increase of the corresponding statistic are more (less) likely and therefore provide evidence for (against) the corresponding micro-mechanism. For instance, a positive value associated to the reciprocity statistic suggests a preferential tendency to reciprocate existing ties.

One intuitive interpretation of the probabilities in (1) and (2) derives from random utility theory (Train, 2009), according to which - given a set of feasible alternatives - individuals choose the course of action yielding the highest level of utility. When nodes get an opportunity for change, they choose the tie in the one-mode (i.e., they change the composition of the their portfolios of external partners) or in the two-mode network (i.e, they change the composition of their portfolios of internal activities) in a way that is expected to produce the highest level of expected utility. Because network nodes can only change ties under their direct control and are only interested in the immediate return deriving from the change, actors in SAOMs are, at best, myopic (or "local") optimizers (Snijders, 2005). The utility function in SAOMs is defined as the sum of the evaluation function and a random term which is assumed to be distributed as a Type I Extreme distribution (Luce and Suppes, 1965; McFadden, 1973) leading to the multinomial probabilities in (1) and (2).

A detailed formulation of the model and the parameter estimation can be found in Snijders (2001) and Snijders et al. (2013). 


\subsection{Model evaluation}

Evaluating the fit of an estimated SAOM - and mechanisms-oriented network models more generally (Snijders and Steglich, 2015) - involves assessing how well the model is able to reproduce salient characteristics of the observed networks that are not explicitly modeled (Amati et al., 2018). Statistics associated to these characteristics are referred to as auxiliary statistics. The most widely used auxiliary statistics involve characteristics of the indegree and the outdegree distributions. Outdegree and indegree distributions describe the connectivity of nodes and therefore are constitutive structural features of the network.

Following the approach developed for exponential random graph models by Hunter et al. (2008), the goodness of fit can be evaluated using continuous-time simulation of the network co-evolution trajectories from the estimated model. More specifically, the distribution of the auxiliary statistics is computed given a large number of simulations based on the estimates. For the model to fit the data well, the observed value of the statistics should not be extreme in this distribution.

A statistical test for the goodness of fit of SAOMs was recently developed by Lospinoso (2012), who proposed to use a Monte Carlo test based on Mahalanobisdistance. The test involves simulating a very large number of co-evolutionary trajectories of the one-mode and two-mode networks, and comparing the average values of the simulated auxiliary statistics to the corresponding values that are actually observed. The model fits the data well when the null hypothesis that simulated and actual values are the same cannot be rejected, i.e., when actual and simulated values are sufficiently close. Closeness of the observed values and the average of the 
simulated values is measured using the Mahalanobis distance. Formally, let $\bar{S}^{*}$ be the vector of auxiliary statistics, $s^{*}$ the values observed in the data and $\Sigma_{S^{*}}$ the covariance matrix of the auxiliary statistics. Then, the test statistic is defined as

$$
D=\left(\bar{S}^{*}-s^{*}\right)^{T} \Sigma_{S^{*}}^{-1}\left(\bar{S}^{*}-s^{*}\right)
$$

The empirical cumulative distribution function of $D$ computed using the simulations is then used as a basis for inference.

\section{Empirical illustration}

\subsection{Setting}

We use relational and attributional data collected on all the hospitals operating within the regional health care system of Abruzzo, a small geographical region in Central Italy with a resident population of approximately 1.3 million people. The Italian national health system is a publicly funded health care system providing universal coverage to all citizens. Through the ministry of health, the national government controls the distribution of tax revenue for publicly financed health care, and defines a national minimum statutory benefits package to be offered to all citizens - the so called essential care. The Italian national health system follows a federal model with the regions in the country responsible for managing, organizing and delivering health care services at the local level.

Regional health care systems are internally partitioned into non-overlapping local health units (LHU) whose mandate is to make health care services generally available 
and accessible across the regional territory. Homogenous coverage is particularly important for regions like Abruzzo where communities living in mountain areas may be difficult to reach. The six LHUs in which the region is partitioned, represent the natural reference markets for hospitals and are of similar size in terms of the resident population they serve.

Data were made available by the regional health agency. Interorganizational networks are reconstructed on the basis of interhospital patient referral flows observed during the period 2003-2011. Referral relations involve the transfer of patients on the basis of the decision taken by a hospital ("sender") to involve a partner hospital ("receiver") in the collaborative solution of a clinical case. We focus on the transfer of elective patients (i.e., patients that are not critically ill and hence are not classified as emergency patients). For this category of patients the sender hospital may typically choose among multiple potential partners on the basis of a variety of operational (e.g., available capacity), locational (e.g., proximity), and medical (e.g., clinical competencies) consideration. In the case of elective patients, relations between hospitals follow clinical decisions and are activated in the interest of the patient, rather than following bilateral contractual arrangements. As explained in Kitts et al. (2017), patient referral decisions are hospital-level decisions with both clinical as well as economic implications.

For each hospital, the composition of internal portfolio of clinical specialties was coded as a 2-mode network affiliating hospitals to their internal activities, defined in terms of organizational units representing specialized pools of clinical and medical knowledge. Detailed yearly information on organizational attributes was also made 
available.

Thirty-five public and private accredited hospitals were in operation in Abruzzo during the observation period. This number remained relatively stable over time with no new hospitals appearing, and five existing small hospitals ceasing operations since 2009. Clinical specialties represent activities for hospitals - pools of specialized medical knowledge organized within distinct organizational units. Examples include cardiology, immunology and neurology. While the distribution of clinical specialties across hospitals changed (with hospitals adding or dropping specialties), the aggregate number of clinical specialties available at the regional level remained stable over time. The set of clinical activities collectively maintained by hospitals may be understood as the stock of medical knowledge available to the regional population.

\subsection{Data}

Figure 3 depicts the one-mode network at the beginning of the observation period (2003). Red circles represent the hospitals and the ties depict the collaborative referral relations among them. In Figure 3, the network diagram is superimposed to the geographical map of the geographical region where the hospitals are located. Position of the nodes corresponds to the location of the hospitals.

- Figure 3 about here -

Over the period of observation, 385 new collaborative referral relations were established across all hospitals and 364 existing collaborative referral relations dissolved. Stability between consecutive observations for the patient transfer network 
is measured by Jaccard coefficients (Snijders et al., 2010), ranging from 0, if all ties change, to 1 , if all ties remain the same in successive time periods. The stability of collaborative networks is relatively high as revealed by Jaccard coefficients ranging between 0.46 and 0.58 . The first block of Table A1 summarizes the changes in the observed one-mode network in more detail.

The two-mode network data specify the internal portfolios of organizational activities, i.e., how individual hospitals are associated with the 46 clinical specialties (or activities) present in the region. The composition of the portfolio of clinical activities defines the niche of hospital - i.e., the sub-space that it occupies in the overall space of medical knowledge. Hospitals with wider niches like, for example, general hospitals, contain many clinical specialties. Because hospital management is responsible for economic performance, hospitals have at least some discretion over the composition of their internal portfolio of clinical activities and their internal organization.

One of example of such hospital is the Ospedali Riuniti SS Annunziata, located in Chieti, whose internal portfolio included 32 of the 46 clinical specialties present in the region at the end the last year of observation. Specialist hospitals manage more restricted portfolios of clinical activities, and occupy, therefore, narrower niches. For example, the Casa di Cura San Francesco in the city of Vasto specializes exclusively in physical rehabilitation activities, and provides no other care services. In more general terms, the internal composition of portfolios of clinical activities determines how medical knowledge is distributed within the organizational community, and where it is actually located within the region. 
Figure 4 illustrates the distribution of activities across hospitals at the beginning of the observation period (2003). The blue rectangles represent the clinical specialties. The width of each rectangle is proportional to the number of hospitals having that specialty (the "indegrees" of the 2-mode network). Specialties number 15 (general medicine) and 4 (general surgery) are the most popular, i.e., they are present in almost every hospital in the region. The red rectangles represent the hospitals. The width of each rectangle is proportional to the size of the portfolio of each hospital (the "outdegrees" in the 2-mode network). The largest hospital in terms of scope of internal activities is the Ospedale Civile dello Spirito Santo of Pescara (id code 130018). The stability of the two-mode network is very high as revealed by the number of newly created (182) and severed ties (160), and by Jaccard coefficients ranging between 0.81 and 0.96 (Table A1).

Table A1 and Table A2 in Appendix A provide detailed information concerning network changes and some basic network statistics for both the one-mode and the two-mode networks.

- Figure 4 about here -

\subsection{Empirical model specification}

We now provide a brief description of the effects included in the models that we estimate in the empirical part of the study. We start by considering the effects associated with change in the one-mode network. The simplest effects are outdegree and reciprocity. The former describes the propensity of organizations to establish ties, whereas the latter to reciprocate existing incoming ties. More complex effects 
involve more than two nodes.

Generalized transitivity represents a closure mechanism whereby ties are formed to close multiple two-paths. In generalized transitivity, organizations tend to create ties to partners of their partners.

The interaction between reciprocity and generalized transitivity effect captures the tendency of organizations to form reciprocated ties embedded in transitive configurations of ties - an effect that is typically predicted to be negative (Block, 2015).

The outdegree and indegree centralization effects and the out-in degree assortativity effect model the outdegree and indegree distributions and their correlation. Therefore, they model types of preferential attachment mechanisms. More specifically, they describe the tendency for (i) more active organizations to establish more ties (outdegree centralization); (ii) organizations to choose more popular organizations as partners (indegree centralization), and (iii) more active organizations to choose more popular organizations (out-in degree assortativity).

We introduce now the effects modeling the evolution of the two-mode network. Baseline effects are the outdegree, modeling the tendency of organizations to own activities, and the 4-cycle, describing the preferential tendency of organizations to share multiple activities. Other effects used to capture the outdegree and the indegree distributions are: (i) the outdegree truncated at two effect, controlling for the tendency of organizations to maintain only 1 activity and thus to be specialist hospitals; (ii) the organizational scope effect, modeling the tendency of generalist organizations (i.e., organizations holding a large portfolio of activities) to increase further - or maintain at a high level - the number of specialties they control; (iii) 
the activity diffusion effect modeling the tendency of organizations to have activities in their portfolio that are also in the portfolio of many other organizations; (iv) the out-in degree assortativity effect controlling for the tendency of larger generalist organizations to maintain in their internal portfolios activities that are relatively common in the community, i.e., activities present in many other hospitals. This effect also describes a systematic differentiation in the frequency distribution of activities in the sense that some activities will be held by many organizations, while other activities will be held by few; (v) the anti-in-isolates, and the (vi)anti-in-near isolates effects modeling the tendency of organizations to maintain activities that are not in the portfolio of any other organization, or are in the portfolio of only one other organization, respectively. Together, these latter effects are included to control for features of the data generated by known tendencies of organizations within a field to become progressively more differentiated over time.

Multilevel mixing and closure are the effects of central interest in the analysis. The corresponding multilevel mechanisms are represented by statistics in the evaluation function of one network that are dependent on the ties in the other network. The first set of multilevel effects (mixing) describe how the internal structure of the organizations (ties in the two-mode network) affects interorganizational relations (ties in the one-mode network). Focal closure is the tendency of hospitals to form ties with partners having the same clinical activities. The scope on outdegree effect describes the tendency of generalist organizations to increase the number of referral partners. In our specific context, the tendency of hospitals with many clinical specialties to rely on many partner hospitals. The second set of multilevel effects 
(closure) describe how (one-mode) ties in the interorganizational network affect the internal structure of the organizations (ties in the two-mode network). Membership closure reprsents the tendency of connected organizations to become more similar in terms of their internal activities. The outdegree on scope effect describes how organizations with many interorganizational relations tend to increase the number of specialties (and hence to become more generalist).

Table 1 summarizes our discussion of the network effects included in the model by reporting, for each effect, an intuitive graphical representation and a brief verbal description of the underlying mechanism. We refer readers interested in the mathematical definitions of the effects to Table B1 in Appendix B and to Snijders et al. (2010) and Snijders et al. (2013).

- Table 1 about here -

Obviously, endogenous network mechanisms may not be the only factors affecting change in interorganizational networks and in the internal structure of organizational nodes. For this reason, the empirical model specification includes a number of dyadic and monadic (organization-specific) covariates that prior studies have found to affect the propensity of organizations to collaborate (Kitts et al., 2017; Stadtfeld et al., 2016).

Geographical distance is a dyadic covariate measured as the (logarithm of the) driving time between any two hospitals in the data, and is included to control for the known tendency of hospitals to refer patients to more proximate partners (other conditions being equal). Driving time is preferred to physical distance because the mountain ranges that cover the majority of the regional area make physical distance 
less useful than driving time as a measure of effective distance between organizations.

The remaining organization-specific covariates enter the empirical model specification as sender, receiver and homophily effects to capture additional factors that may affect interorganizational collaboration.

Urban location is a dichotomous variable taking the value 1 for hospitals located in major urban areas and 0 otherwise. Urban location is included to control for the tendency of hospitals located in rural and in small urban communities to refer patients to hospitals located in metropolitan areas.

LHU membership is a categorical variable that captures joint membership of hospitals in the different local health units (LHUs) in which the region is partitioned for administrative purposes. Hospitals in the same LHU are more likely to collaborate because they belong to the same superordinate administrative and managerial structure.

Institutional form is a categorical variable that records the different institutional profiles of hospitals in the region. The institutional form of a hospital affects its governance structure and stated objectives, but it also constrains the activities and services that may be provided. We identified three alternative institutional forms. The first involves public hospitals owned directly by the LHU (labeled LHU hospital) providing secondary care services. The second form contains university polyclinics or hospitals that are qualified to render highly specialized (or "tertiary"') services, and research activities. The third form includes private accredited hospitals - hospitals controlled by private owners that are eligible to provide secondary care services under the single payer system. We control for institutional form to account for 
the tendency of hospitals to refer patients to partners with compatible institutional profiles.

The hospitals' occupancy rate is included to account for capacity utilization and for the possibility that interhospital patient flows be affected by differences in available capacity between partner hospitals. The closer the hospital occupancy rate is to 1 , the closer the hospital is to its full capacity. While certain hospitals can operate, occasionally, above their full capacity, they cannot do so indefinitely.

It is common for hospitals in public health care systems with a single payer to receive standard cost-based reimbursements according to DRG (diagnosis-related grouping) tariffs decided at the national level. Average patient value captures the average monetary value of resources deployed by hospital to treat their patients, i.e., the average cost of treatment per discharged patient. The variable is included to control for the possibility that interhospital patient relations simply reflect differences in costs of treatment. A summary and basic descriptive statistics of the monadic and dyadic covariates is reported in Table A3 in Appendix A.

\subsection{Results}

The model parameters discussed in this section are estimated by the method of moments, computed using the stochastic approximation algorithm (Snijders, 2001) implemented in the R library RSiena (Ripley et al., 2018). To simplify exposition, in Table 2 we reported only the results of more direct interest to our discussion.

The complete model that includes an additional set of rate parameters, and the exogenous covariate effects is reported in Table $\mathrm{C} 1$ in Appendix $\mathrm{C}$ where the 
estimates are briefly discussed.

The template of the R-script that we have adapted to produce the estimates reported in this section is available at https://www.stats.ox.ac.uk/ snijders/ siena/RscriptSienaBipartite.R.

Table 2 reports the estimates of the parameters in the evaluation functions controlling change in the one-mode and two-mode networks. The table is organized in blocks of parameters pertaining to conceptually different aspects of the model. Parameters in block $1 M$ are associated with endogenous mechanisms that shape the structure of the interorganizational (1-mode) network. Parameters in block $2 M$ of Table 2 are associated with endogenous mechanisms that regulate processes of internal organizational change - i.e., change in the two-mode network of organizationsby-activities. Finally, block $M L$ contains the effects of multilevel mechanisms that are of central interest.

For simplicity, model parameters are interpreted individually. In network models this interpretation is partially misleading because (i) many of the effects operate simultaneously; (ii) the effects are the outcome of social mechanisms that are concatenated, and (ii) the parameter estimates depend, in part, on the initial state of the system that is itself not modeled (Koskinen et al., 2015).

Both in the one-, as well as the two-mode networks the outdegree parameters play the role of intercepts. Their negative sign reflects the sparsity (low density) of the corresponding networks. Jointly considered, estimates of endogenous network effect (reported in block $1 M$ ) provide evidence of preferential tendencies toward reciprocity (reciprocity) and clustering based on transitive closure (generalized transitivity). 
The negative value of the parameter related to the interaction between reciprocity and generalized transitivity indicates that the tendency to reciprocate is smaller between hospitals embedded in transitive triplets. We note that the coefficient for this interaction effect is smaller in absolute value than both main effects.

Evidence of centralization tendencies based on in- and outdegree is also found (in- and outdegree centralization). We note a tendency towards disassortativity in the referral network: hospitals with many partners preferentially select as partners hospitals with fewer partners (out-in degree assortativity).

The estimates of the network effects on the portfolio of internal organizational activities are reported in block $2 M$. The 4-cycle effect suggests a tendency of organizations sharing a common specialty, to share additional specialties or to maintain the specialties they both have. In either case, the 4-cycle effect reveals a general tendency of collaborating hospitals to be, or become, more similar - relational processes consistent with processes of institutional isomorphism (DiMaggio and Powell, 1983).

The outdegree truncated at two effect is negative suggesting a positive tendency toward the maintenance (or creation) of single-specialty hospitals, or - in other words - a tendency of specialist hospitals to remain specialists.

The positive effect of organizational scope indicates the tendency of larger organizations to increase their scope and size further or to maintain their larger scope and size. In other words, generalist hospitals tend to exploit size-based advantages by maintaining or further expanding their internal portfolio of activities.

The significantly positive activity diffusion effect reveals a tendency of organi- 
zations in the community to conform to the choices of internal activities made by other organizations. The aggregate effect of these micro-processes is that organizations in the community tend to become progressively more similar - a conclusion consistent with institutionalist perspectives on interorganizational communities and fields (DiMaggio and Powell, 1983).

The negative estimate of the anti in-isolates effect parameter suggests a tendency toward the progressive disappearance from internal organizational portfolios of activities that are held only by one hospital, i.e., of activities for which demand in the community is weak.

The negative value of the parameter of the anti in-near-isolates effect reveals a tendency of organizations in the sample to abandon activities that will then be held by only one other organization - i.e., to progressively drop from their internal portfolios clinical activities that are uncommon.

Finally, the tendency against out-in assortativity in the 2-mode network suggests that hospitals with larger portfolios of activities tend to maintain clinical specialties that are relatively uncommon in the community.

Estimates associated with the multilevel mechanisms of interest are reported in block $M L$ at the bottom of the table. Concerning the closure mechanisms, we note that only focal closure is significant. The corresponding positive coefficient indicates that hospitals holding similar portfolios of internal activities display a preferential tendency to develop collaborative relations. Membership closure has no significant effect: hospitals connected by collaborative patient referral relations do not necessarily develop similar portfolios of internal activities. Jointly considered, 
the multilevel closure effects suggest a clear dominance of social selection over social influence processes as closure-generating mechanism in our data.

The multilevel mixing effects are both significant, but in opposite directions. The positive coefficient of the scope on outdegree effect suggests that hospitals managing larger portfolios of activities tend to increase the size of their network of partners. In other words, the estimates reveal a tendency toward multilevel assortativity. The negative coefficient of the outdegree on scope effect indicates that hospitals with fewer partners tend to increase the size of their portfolios of internal organizational activities. The former multilevel mixing effect suggests that organizations with diversified portfolios of internal activities are more capable of managing larger networks of partners - an interpretation consistent with "absorptive capacity" arguments (Cohen and Levinthal, 2000). The latter mixing effect suggests that acquisition of internal resources may be a response to difficulties in finding valuable exchange partners.

- Table 2 about here -

\subsection{Model evaluation}

To what extent is the fitted model consistent with the organization and network structures that were actually observed? To address this question, we simulated a large number (3000) of one-mode/two-mode co-evolution trajectories, and computed the value of the auxiliary statistics for each simulated trajectory. We then compare the statistics computed on the simulated networks with the statistics computed on the observed network data. The outcome of this comparison is reported in 
Figure 5 which shows the violin plots of the outdegree and indegree distributions of the one-mode and two-mode networks (Hintze and Nelson, 1998). Each violin plot depicts the distribution (line around the box-plot), the observed count (red dots), the average of the simulated value (black dots) and the outliers (black crosses) for each degree value. The model fits the data well when the distance between red and black dots is small.

Panels $5 \mathrm{a}$ and $5 \mathrm{~b}$ report the distribution of the outdegree and indegree of the (one-mode) interhospital network, respectively. In both cases the model reproduces well the propensity of hospitals to collaborate (panel 5a) and the tendency of hospitals to be selected as partners (panel 5b). The model also reproduces with high fidelity the distribution of internal clinical activities, i.e. the organizational design of the hospitals in the community (Panel 5c). Finally, panel $5 \mathrm{~d}$ shows that the distribution of medical knowledge in the region is also well captured by the multilevel model. The p-values associated with the Mahalanobis distance test reported at the bottom of each panel, demonstrate that the null hypothesis that the simulated networks given the estimated model and the observed networks have similar structural characteristics cannot be rejected.

The post-hoc simulation analysis produced solid evidence that the estimates of the multilevel model reproduce with considerable accuracy: (i) the internal structure of the organizational nodes; (ii) the observed structure of the interorganizational network, and (iii) the distribution of available medical knowledge within the organizational field. The results of the analysis show that the model is capable of explaining data observed at very different structural levels. This conclusion is 
strengthened further by the goodness of fit test based on the triad census and the shortest path length (geodesic distance) auxiliary statistics. As both visual inspection and the Mahalanobis distance test indicate, the model fits well each one of the sixteen triadic configurations of network ties observed in the directed hospital referral network, and almost perfectly the distribution of the shortest path observed among the organizations in the sample (Figure D1 in Appendix D).

- Figure 5 about here -

\section{Discussion and Conclusions}

The view of organizations as hierarchical multilevel social systems articulated by H.A. Simon (1962) continues to inspire contemporary organizational research (Levinthal and Workiewicz, 2018). A new generation of statistical models for multilevel networks is opening new possibilities for exploring and expanding the scope of this classic view. According to Moliterno and Mahony (2011, p.443): "Given that organizations are multilevel systems, a network theory of the organization should, by definition, be multilevel in its scope, considering how networks at one level of the organizational system influence networks at higher and/or lower levels." In this paper we responded to this call to forward multilevel organizational research in the context of interorganizational networks.

We identified the problem of change as central to our understanding of multilevel organizational systems. Representing how networks of relations change among social actors that are themselves changing, remains a prime theoretical and empirical challenge in our understanding of emergent organizational phenomena (Kozlowski 
et al., 2013; Padgett and Powell, 2012; Powell et al., 2005). Taking on this challenge requires the development of a new approach to the analysis of multilevel networks that recognizes change in the internal structure of organizational nodes as both an antecedent and a consequence of change in the structure of interorganizational networks in which organizations are embedded (Moliterno and Mahony, 2011). We know of no available analytical framework that can be readily adopted to represent change happening simultaneously within and between organizations. More specifically, we know of no analytical framework capable of discriminating among alternative theoretical mechanisms of network change that operate across multiple levels to shape the co-evolution of organizational and network structure.

In this study, we have proposed and tested a principled analytical framework that specifies multiple mechanisms coupling change within and between organizations. According to the model, two sets of multilevel mechanisms couple decisions taking place at different structural levels. Consistent with assumptions of stochastic actororiented models for network dynamics (Snijders et al., 2010), the first set involves decisions to change the composition of portfolios of internal organizational activities. The second set involves decisions to change the composition of portfolios of external partners.

We presented a realistically complex case study of interorganizational relations in the field of health care - a field in which internal organizational design decisions are intimately related to the external resources that organizations can access through networks of external partners (Gittell and Weiss, 2004; Lomi and Pallotti, 2012). Our empirical analysis of a regional community of hospital organizations connected 
by collaborative patient referral relations reveals that focal closure dominates membership closure: organizations with similar portfolios of internal activities are more likely to collaborate. Collaborating organizations are not more likely than chance to change their internal structure in the direction of becoming more similar. This result sheds new light on a major ambiguity latent in studies of interorganizational fields inspired by institutional theories (DiMaggio, 1986). Our results clearly indicate that the tendency of interdependent organizations in our sample to be (or become) similar - or mimetic isomorphism - is generated by social selection rather than social influence, contrary to what the majority of studies about diffusion of strategies and practices within interorganizational fields typically assume. To the extent that mimetic isomorphism may be interpreted as a consequence of how organizations respond to uncertainty (DiMaggio and Powell, 1983), the results of our study suggest that organizations in our sample manage uncertainty by establishing network ties with structurally similar partners, rather than imitating current partners that are partially dissimilar.

The results of the study are obviously specific to the sample of organizations that we have selected for study. The setting of the study also has a number of institutional idiosyncrasies. But the model we have presented has broad potential application both in the study of interpersonal, as well as interorganizational networks. Examples of future work that the model might inspire include - but are not limited to: studies of the co-evolution of social relations and cultural tastes (Lewis and Kaufman, 2018), friendship choice and employment preference (Kilduff, 1990), advice networks among managers and words they use to describe their company 
(Lomi et al., 2017). At a more macro level, future studies may find it useful to examine alternative multilevel closure mechanisms in the context of alliance formation between companies with complementary or compatible internal resources (Mitsuhashi and Greve, 2009). Our analysis of multilevel mixing mechanisms reveals new connections between organizational niche width and positions in interorganizational networks. This result may open new perspectives for future research on resource partitioning (Carroll et al., 2002), organizational niches (Podolny et al., 1996), and for processes of resource transfer from generalist to specialist organizations (Carroll and Swaminathan, 2000).

More generally, the model for multilevel networks that we have discussed affords considerable flexibility in the choice of levels of analysis. For example, Lomi and Stadtfeld (2014) apply it to study the co-evolution of advice ties among MBA students and their employment preferences. Fujimoto et al. (2018) adopt it to study the co-evolution of friendship network and participation in sport activities among adolescents. Milewicz et al. (2018) adopt the model to examine non-trade agendas and trade agreements among countries. These works demonstrate the considerable flexibility of the model that may be applied meaningfully across very different levels of analysis within - and now also between organizations.

Two main limitations of the study deserve particular mention as they suggest ways in which future research may improve our understanding of multilevel processes of change within and between organizations. The first limitation is related to the fact that the two component decision processes that define the model are observed during the same time frame. Given a fixed observation window, "slower" decision 
processes will produce fewer observations on change than "faster" decision processes. How severe this problem might be depends on the "internal time clocks" that regulate processes of organizational and network change (Amburgey et al., 1993). In our specific case, for example, the "change rates" estimated from data suggest that change in the composition in the internal portfolios of activities unfolds at a much slower rate than change in the composition of portfolios of external partners (see table $\mathrm{C} 1$ in Appendix C). General considerations of organizational inertia make this result unsurprising (Hannan and Freeman, 1984). But these differences in rates of change imply that fewer observations are available on organizational change than on network change - giving rise to difficulties due to differences in statistical power between sub-processes. While we are not aware of obvious solutions to this "multiple clocks" problem in models based on network panel data, we suspect that future research may produce considerable insight by modeling the change rates directly. Doing so might lead to a more detailed understanding of the factors that slow or, as the case may be, accelerate organizational and network change.

The second limitation inheres in the way we have represented organizational change as a sequence of episodic, discrete changes in the composition of internal portfolios of activities. But organizational change also involves equally important higher-frequency micro-level changes in processes, practices and routines that do not necessarily reflect themselves in observable modifications in the formal composition of internal organizational activities (March, 1981; Rerup and Feldman, 2011). The data we have collected do not allow us to say much about these important sources of organizational change. For similar reasons, the data we have analyzed in this 
paper are probably not appropriate to study process of organizational learning another major determinant of organizational change (Levitt and March, 1988). We suspect that an analysis of continuous processes of internal organizational learning and change may be better supported by research design that produce continuous time, event-oriented observations (Amati et al., 2019).

Despite these limitations, the study remains one of the few available that have specified the co-evolutionary mechanisms coupling change in internal organizational activities and external network structure, identified the different effects of such mechanisms across multiple levels of organizational analysis, and adjudicated their relative empirical significance. The ability of the model to reproduce the aggregate field-level structures that are actually observed starting from assumptions about micro-mechanisms of local dependence operating across levels of analysis, is the central achievement of the new multilevel network approach that we have proposed. 


\section{References}

Ahuja, G., Soda, G., and Zaheer, A. (2012). The genesis and dynamics of organizational networks. Organization science, 23(2):434-448.

Amati, V., Lomi, A., and Mascia, D. (2019). Some days are better than others: Examining time-specific variation in the structuring of interorganizational relations. Social Networks, 57:18-33.

Amati, V., Lomi, A., and Mira, A. (2018). Social network modelling. Annual Review of Statistics and Its Application, 5(1).

Amburgey, T. L., Kelly, D., and Barnett, W. P. (1993). Resetting the clock: The dynamics of organizational change and failure. Administrative Science Quarterly, 38:51-73.

Barnett, W. P. and Carroll, G. R. (1987). Competition and mutualism among early telephone companies. Administrative Science Quarterly, pages 400-421.

Barnett, W. P. and Carroll, G. R. (1995). Modeling internal organizational change. Annual review of sociology, 21(1):217-236.

Baum, J. A., Shipilov, A. V., and Rowley, T. J. (2003). Where do small worlds come from? Industrial and Corporate change, 12(4):697-725.

Beckman, C. M. and Haunschild, P. R. (2002). Network learning: The effects of partners' heterogeneity of experience on corporate acquisitions. Administrative science quarterly, 47(1):92-124. 
Block, P. (2015). Reciprocity, transitivity, and the mysterious three-cycle. Social Networks, 40:163-173.

Block, P., Koskinen, J., Hollway, J., , C., and Stadtfeld, C. (2018). Change we can believe in: comparing longitudinal network models on consistency, interpretability and predictive power. Social Networks, 52:180-191.

Boda, Z. (2018). Social influence on observed race. Sociological Science, 5:29-57.

Borgatti, S. P. and Foster, P. C. (2003). The network paradigm in organizational research: A review and typology. Journal of management, 29(6):991-1013.

Brass, D. J., Galaskiewicz, J., Greve, H. R., and Tsai, W. (2004). Taking stock of networks and organizations: A multilevel perspective. Academy of management journal, 47(6):795-817.

Carroll, G. R., Dobrev, S. D., and Swaminathan, A. (2002). Organizational processes of resource partitioning. Research in organizational behavior, 24:1-40.

Carroll, G. R. and Swaminathan, A. (2000). Why the microbrewery movement? organizational dynamics of resource partitioning in the us brewing industry. American journal of sociology, 106(3):715-762.

Cohen, W. M. and Levinthal, D. A. (1994). Fortune favors the prepared firm. Management science, 40(2):227-251.

Cohen, W. M. and Levinthal, D. A. (2000). Absorptive capacity: A new perspective on learning and innovation. In Strategic Learning in a Knowledge economy, pages 39-67. Elsevier. 
Conaldi, G., Lomi, A., and Tonellato, M. (2012). Dynamic models of affiliation and the network structure of problem solving in an open source software project. Organizational Research Methods, 15(3):385-412.

Contractor, N. S., Wasserman, S., and Faust, K. (2006). Testing multitheoretical, multilevel hypotheses about organizational networks: An analytic framework and empirical example. Academy of Management Review, 31(3):681-703.

Denrell, J. (2003). Vicarious learning, undersampling of failure, and the myths of management. Organization Science, 14(3):227-243.

DiMaggio, P. (1986). Structural analysis of organizational fields: A blockmodel approach. Research in organizational behavior.

DiMaggio, P. and Powell, W. W. (1983). The iron cage revisited: Collective rationality and institutional isomorphism in organizational fields. American sociological Review, 48(2):147-160.

Easley, D. and Kleinberg, J. (2010). Networks, crowds, and markets: Reasoning about a highly connected world. Cambridge University Press.

Fujimoto, K., Snijders, T. A. B., and Valente, T. W. (2018). Multivariate dynamics of one-mode and two-mode networks: Explaining similarity in sports participation among friends. Network Science, pages 1-26.

Gibbons, R. and Henderson, R. (2012). Relational contracts and organizational capabilities. Organization Science, 23(5):1350-1364. 
Gittell, J. H. and Weiss, L. (2004). Coordination networks within and across organizations: A multi-level framework. Journal of Management Studies, 41(1):127-153.

Greve, H. R. (2005). Interorganizational learning and heterogeneous social structure. Organization Studies, 26(7):1025-1047.

Gulati, R. (1999). Network location and learning: The influence of network resources and firm capabilities on alliance formation. Strategic management journal, 20(5):397-420.

Hannan, M. T. and Freeman, J. (1984). Structural inertia and organizational change. American sociological review, pages 149-164.

Hintze, J. L. and Nelson, R. D. (1998). Violin plots: a box plot-density trace synergism. The American Statistician, 52(2):181-184.

Hitt, M. A., Beamish, P. W., Jackson, S. E., and Mathieu, J. E. (2007). Building theoretical and empirical bridges across levels: Multilevel research in management. Academy of Management journal, 50(6):1385-1399.

Hollway, J., Lomi, A., Pallotti, F., and Stadtfeld, C. (2017). Multilevel social spaces: The network dynamics of organizational fields. Network Science, 5(2):187-212.

Hunter, D. R., Goodreau, S. M., and Handcock, M. S. (2008). Goodness of fit of social network models. Journal of the American Statistical Association, 103(481):248-258.

Kilduff, M. (1990). The interpersonal structure of decision making: A social com- 
parison approach to organizational choice. Organizational behavior and human decision processes, 47(2):270-288.

Kitts, J. A., Lomi, A., Mascia, D., Pallotti, F., and Quintane, E. (2017). Investigating the temporal dynamics of interorganizational exchange: Patient transfers among italian hospitals. American Journal of Sociology, 123(3):850-910.

Kogut, B. and Walker, G. (2001). The small world of germany and the durability of national networks. American sociological review, pages 317-335.

Koskinen, J., Caimo, A., and Lomi, A. (2015). Simultaneous modeling of initial conditions and time heterogeneity in dynamic networks: An application to foreign direct investments. Network Science, 3(1):58-77.

Kozlowski, S. W., Chao, G. T., Grand, J. A., Braun, M. T., and Kuljanin, G. (2013). Advancing multilevel research design: Capturing the dynamics of emergence. Organizational Research Methods, 16(4):581-615.

Kozlowski, S. W. and Klein, K. J. (2000). A multilevel approach to theory and research in organizations: Contextual, temporal, and emergent processes. In Klein, K. J. and Kozlowski, S. W., editors, Multilevel theory, research, and methods in organizations: Foundations, extensions, and new directions. Jossey-Bass.

Levinthal, D. A. and Workiewicz, M. (2018). When two bosses are better than one: Nearly decomposable systems and organizational adaptation. Organization Science, 29(2):207-224. 
Levitt, B. and March, J. G. (1988). Organizational learning. Annual review of sociology, 14(1):319-338.

Lewis, K. and Kaufman, J. (2018). The conversion of cultural tastes into social network ties. American Journal of Sociology, 123(6):1684-1742.

Lomi, A. and Pallotti, F. (2012). Relational collaboration among spatial multipoint competitors. Social networks, 34(1):101-111.

Lomi, A. and Pallotti, F. (2013). How to close a hole: Exploring alternative closure mechanisms in interorganizational networks. In Lusher, D., Koskinen, J., and Robins, G., editors, Exponential Random Graph Models for Social Networks: Theory, Methods and Applications, pages 202-212. Cambridge University Press: Cambridge, UK.

Lomi, A. and Pattison, P. (2006). Manufacturing relations: An empirical study of the organization of production across multiple networks. Organization science, $17(3): 313-332$.

Lomi, A., Robins, G., and Tranmer, M. (2016). Introduction to multilevel social networks. Social Networks, 44:266-268.

Lomi, A. and Stadtfeld, C. (2014). Social networks and social settings: Developing a coevolutionary view. KZfSS Kölner Zeitschrift für Soziologie und Sozialpsychologie, 66(1):395-415.

Lomi, A., Tasselli, S., and Zappa, P. (2017). The network structure of organizational 
vocabularies. In Structure, Content and Meaning of Organizational Networks: Extending Network Thinking, pages 65-85. Emerald Publishing Limited.

Lorenzoni, G. and Lipparini, A. (1999). The leveraging of interfirm relationships as a distinctive organizational capability: a longitudinal study. Strategic Management Journal, pages 317-338.

Lospinoso, J. A. (2012). Statistical models for social network dynamics. PhD thesis, University of Oxford.

Luce, R. and Suppes, P. (1965). Preference, utility, and subjective probability. In Luce, R. D., Bush, R. R., and Eugene, G., editors, Handbook of Mathematical Psychology, volume 3, pages 249-410. New York: Wiley.

March, J. G. (1981). Footnotes to organizational change. Administrative science quarterly, pages 563-577.

Mascia, D., Pallotti, F., and Angeli, F. (2017). Don't stand so close to me: competitive pressures, proximity and inter-organizational collaboration. Regional Studies, 51(9):1348-1361.

McFadden, D. (1973). Conditional logit analysis of qualitative choice behavior. In Zarembka, P., editor, Frontiers in Econometrics, pages 105-142. Academic Press, New York.

Milewicz, K., Hollway, J., Peacock, C., and Snidal, D. (2018). Beyond trade: the expanding scope of the nontrade agenda in trade agreements. Journal of Conflict Resolution, 62(4):743-773. 
Mitsuhashi, H. and Greve, H. R. (2009). A matching theory of alliance formation and organizational success: Complementarity and compatibility. Academy of Management Journal, 52(5):975-995.

Moliterno, T. P. and Mahony, D. M. (2011). Network theory of organization: A multilevel approach. Journal of Management, 37(2):443-467.

Newman, M. E. (2003). Mixing patterns in networks. Physical Review E, 67(2):026126.

Padgett, J. F., Lee, D., and Collier, N. (2003). Economic production as chemistry. Industrial and Corporate Change, 12(4):843-877.

Padgett, J. F. and Powell, W. W. (2012). The emergence of organizations and markets. Princeton University Press.

Pfeffer, J. and Salancik, G. R. (2003). The external control of organizations: A resource dependence perspective. Stanford University Press.

Podolny, J. M., Stuart, T. E., and Hannan, M. T. (1996). Networks, knowledge, and niches: Competition in the worldwide semiconductor industry, 1984-1991. American journal of sociology, 102(3):659-689.

Powell, W. W., Koput, K. W., and Smith-Doerr, L. (1996). Interorganizational collaboration and the locus of innovation: Networks of learning in biotechnology. Administrative science quarterly, pages 116-145.

Powell, W. W., White, D. R., Koput, K. W., and Owen-Smith, J. (2005). Network 
dynamics and field evolution: The growth of interorganizational collaboration in the life sciences. American journal of sociology, 110(4):1132-1205.

Rerup, C. and Feldman, M. S. (2011). Routines as a source of change in organizational schemata: The role of trial-and-error learning. Academy of Management Journal, 54(3):577-610.

Ripley, R. M., Snijders, T. A. B., B'oda, Z., V"or"os, A., and Preciado, P. (2018). Manual for Siena version 4.0. Technical report, Oxford: University of Oxford, Department of Statistics; Nuffield College. R package version 1.2-12. https://www.cran.r-project.org/web/packages/RSiena/.

Robins, G., Pattison, P., and Wang, P. (2009). Closure, connectivity and degree distributions: Exponential random graph $\left(\mathrm{p}^{*}\right)$ models for directed social networks. Social Networks, 31(2):105-117.

Schelling, T. C. (1998). Social mechanisms and social dynamics. Social mechanisms: An analytical approach to social theory, pages 32-44.

Shortell, S. M., Gillies, R. R., Anderson, D. A., Mitchell, J. B., and Morgan, K. L. (1993). Creating organized delivery systems: the barriers and facilitators. Journal of Healthcare Management, 38(4):447.

Simon, H. A. (1962). The architecture of complexity. Proceedings of the American Philosophical Society, 106(6):467-482.

Snijders, T. A. (2001). The statistical evaluation of social network dynamics. Sociological methodology, 31(1):361-395. 
Snijders, T. A. (2005). Models for longitudinal network data. Models and methods in social network analysis, 1:215-247.

Snijders, T. A. (2016). The multiple flavours of multilevel issues for networks. In Multilevel network analysis for the social sciences, pages 15-46. Springer.

Snijders, T. A., Lomi, A., and Torló, V. J. (2013). A model for the multiplex dynamics of two-mode and one-mode networks, with an application to employment preference, friendship, and advice. Social networks, 35(2):265-276.

Snijders, T. A. and Steglich, C. E. (2015). Representing micro-macro linkages by actor-based dynamic network models. Sociological methods $\&$ research, 44(2):222271.

Snijders, T. A., Van de Bunt, G. G., and Steglich, C. E. (2010). Introduction to stochastic actor-based models for network dynamics. Social networks, 32(1):44-60.

Stadtfeld, C., Mascia, D., Pallotti, F., and Lomi, A. (2016). Assimilation and differentiation: A multilevel perspective on organizational and network change. Social Networks, 44:363-374.

Steglich, C., Snijders, T. A., and Pearson, M. (2010). Dynamic networks and behavior: Separating selection from influence. Sociological methodology, 40(1):329-393.

Train, K. E. (2009). Discrete choice methods with simulation. Cambridge university press.

Tranmer, M., Pallotti, F., and Lomi, A. (2016). The embeddedness of organizational 
performance: Multiple membership multiple classification models for the analysis of multilevel networks. Social Networks, 44:269-280.

Tranmer, M., Steel, D., and Browne, W. J. (2014). Multiple-membership multipleclassification models for social network and group dependences. Journal of the Royal Statistical Society: Series A (Statistics in Society), 177(2):439-455.

Wang, P., Robins, G., Pattison, P., and Lazega, E. (2013). Exponential random graph models for multilevel networks. Social Networks, 35(1):96-115.

Wang, P., Robins, G., Pattison, P., and Lazega, E. (2016). Social selection models for multilevel networks. Social Networks, 44:346-362.

Watts, D. J. and Strogatz, S. H. (1998). Collective dynamics of 'smallworld'networks. Nature, 393(6684):440.

Zappa, P. and Lomi, A. (2015). The analysis of multilevel networks in organizations: Models and empirical tests. Organizational Research Methods, 18(3):542-569. 
Tables

\begin{tabular}{|c|c|c|}
\hline Statistic & Representation & Description: Preferential tendency \\
\hline \multicolumn{3}{|c|}{ (1M) One-mode effects } \\
\hline Outdegree & & to establish referral relations \\
\hline Reciprocity & $i \mathrm{O}$ & to reciprocate referral relations \\
\hline $\begin{array}{l}\text { Generalized } \\
\text { transitivity } \\
\text { (gwesp) }\end{array}$ & & $\begin{array}{l}\text { to establish relations with partners connected to the } \\
\text { same third parties }\end{array}$ \\
\hline $\begin{array}{l}\text { Generalized } \\
\text { transitivity } \\
\times \text { reciprocity }\end{array}$ & j & $\begin{array}{l}\text { to establish reciprocated relations with partners con- } \\
\text { nected to the same third parties }\end{array}$ \\
\hline $\begin{array}{l}\text { Outdegree } \\
\text { centralization }\end{array}$ & & $\begin{array}{l}\text { of organizations with high out-degrees to increase their } \\
\text { out-degrees further }\end{array}$ \\
\hline $\begin{array}{l}\text { Indegree } \\
\text { centralization }\end{array}$ & & $\begin{array}{l}\text { to choose partners with high in-degrees, i.e., popular } \\
\text { partners }\end{array}$ \\
\hline $\begin{array}{l}\text { Out-in degree } \\
\text { assortativity }\end{array}$ & $0^{2}$ & $\begin{array}{l}\text { of organizations with many partners (i.e., active or- } \\
\text { ganizations) to establish relations with organizations } \\
\text { already chosen as partners by many others (i.e., pop- } \\
\text { ular organizations) }\end{array}$ \\
\hline \multicolumn{3}{|c|}{ (2M) Two-mode effects } \\
\hline Outdegree & $i \bigcirc \square a$ & maintain clinical activities \\
\hline 4-cycle & $\begin{array}{l}a_{2} \square \bigcirc j \\
i \bigcirc-\square \\
a_{1}\end{array}$ & $\begin{array}{l}\text { of organization already having one clinical activity in } \\
\text { common to increase the number of clinical activities } \\
\text { in common }\end{array}$ \\
\hline $\begin{array}{l}\text { Outdegree } \\
\text { truncated at } 2\end{array}$ & $\begin{array}{ll}i & \square \\
\bigcirc & \square\end{array}$ & to maintain a single internal activity \\
\hline
\end{tabular}




\begin{tabular}{l|lll|l}
$\begin{array}{l}\text { Organizational } \\
\text { scope }\end{array}$ & & & of generalist organizations to increase their scope (i.e., \\
to include additional clinical activities)
\end{tabular}

Table 1: Statistics modeling the co-evolution of a one-mode and a two-mode network. Red circles are health care organizations. Blue squares are clinical activities. Black edges depict referral relations and grey edges denote two-mode relations. Solid edges represent relations existing at time $T_{1}$. Dotted edges represent relations that are observed at $T_{2}$ but not at $T_{1}$. 


\begin{tabular}{l|rl}
\hline & Est. & s.e. \\
\hline \multicolumn{3}{c}{$(1 M)$ One-mode effects } \\
outdegree & -5.826 & $0.436^{* * *}$ \\
reciprocity & 1.650 & $0.294^{* * *}$ \\
general transitivity & 1.531 & $0.194^{* * *}$ \\
reciprocity $\times$ general transitivity & -0.701 & $0.237^{* *}$ \\
indegree - centralization & 0.983 & $0.130^{* * *}$ \\
outdegree - centralization & 0.729 & $0.170^{* * *}$ \\
out-in degree assortativity & -0.318 & $0.077^{* * *}$ \\
\hline \multicolumn{3}{c}{ (2M) Two-mode effects } \\
outdegree & -7.173 & $0.901^{* * *}$ \\
4-cycle & 0.036 & $0.007^{* * *}$ \\
outdegree truncated at 2 & -4.013 & $0.852^{* * *}$ \\
organizational scope & 2.299 & $0.398^{* * *}$ \\
activity diffusion & 1.908 & $0.316^{* * *}$ \\
out-in degree assortativity & -0.814 & $0.162^{* * *}$ \\
anti in-isolates & -0.927 & $0.433^{*}$ \\
anti in-near-isolates & -1.483 & $0.412^{* * *}$ \\
\hline \multicolumn{3}{c}{ focal closure Multilevel effects } \\
scope on outdegree $\mid 0.042$ & $0.012^{* * *}$ \\
membership closure & 0.247 & $0.063^{* * *}$ \\
outdegree on scope & 0.175 & 0.127 \\
\hline
\end{tabular}

${ }^{* * *} p<0.001,{ }^{* *} p<0.01,{ }^{*} p<0.05$

Table 2: Estimated coefficients and corresponding standard errors for the evaluation functions of the one-mode two-mode co-evolution model. The asterisks represent two-sided p-values. 


\section{Figures}

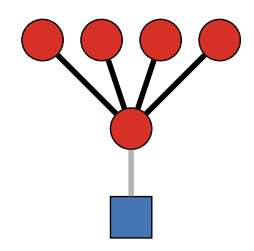

(a)

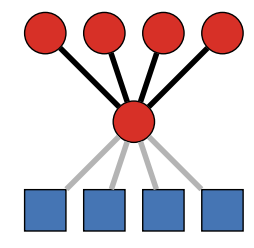

(b)

Figure 1: Multilevel mixing mechanisms. (Red) Circles are organizations. (Blue) Squares are internal activities. Black edges are interorganizational relations. Grey edges are containment relations linking organizations to their internal activities. In Panel (a), a specialist organization (with one internal activity) tends to establish network ties with many exchange partners (multilevel disassortativity). In Panel (b), a generalist organization (holding a large portfolio of internal activities) tends to establish network ties with many exchange partners (multilevel assortativity).

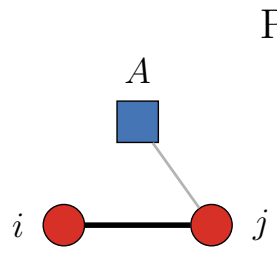

$T 1$

\section{Panel 1}

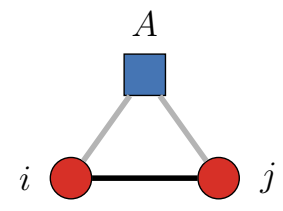

$T 2$

Panel 2

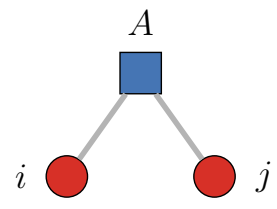

$T 1$

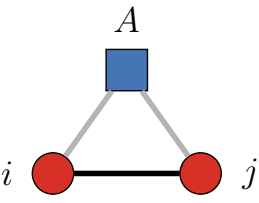

$T 2$

Figure 2: Multilevel closure mechanisms. (Red) Circles are organizations. (Blue) Squares are internal activities. Black edges are interorganizational relations. Grey edges are containment relations linking organizations to their internal activities. In panel 1, organizations $i$ and $j$ are connected at time $T 1$ and tend to become more similar by sharing activity A at time T2 (Multilevel membership closure). In panel 2, organizations $i$ and $j$ hold the same activity $A$ in their internal portfolio at time $T 1$ and tend to become connected at time T2 (Multilevel focal closure). 


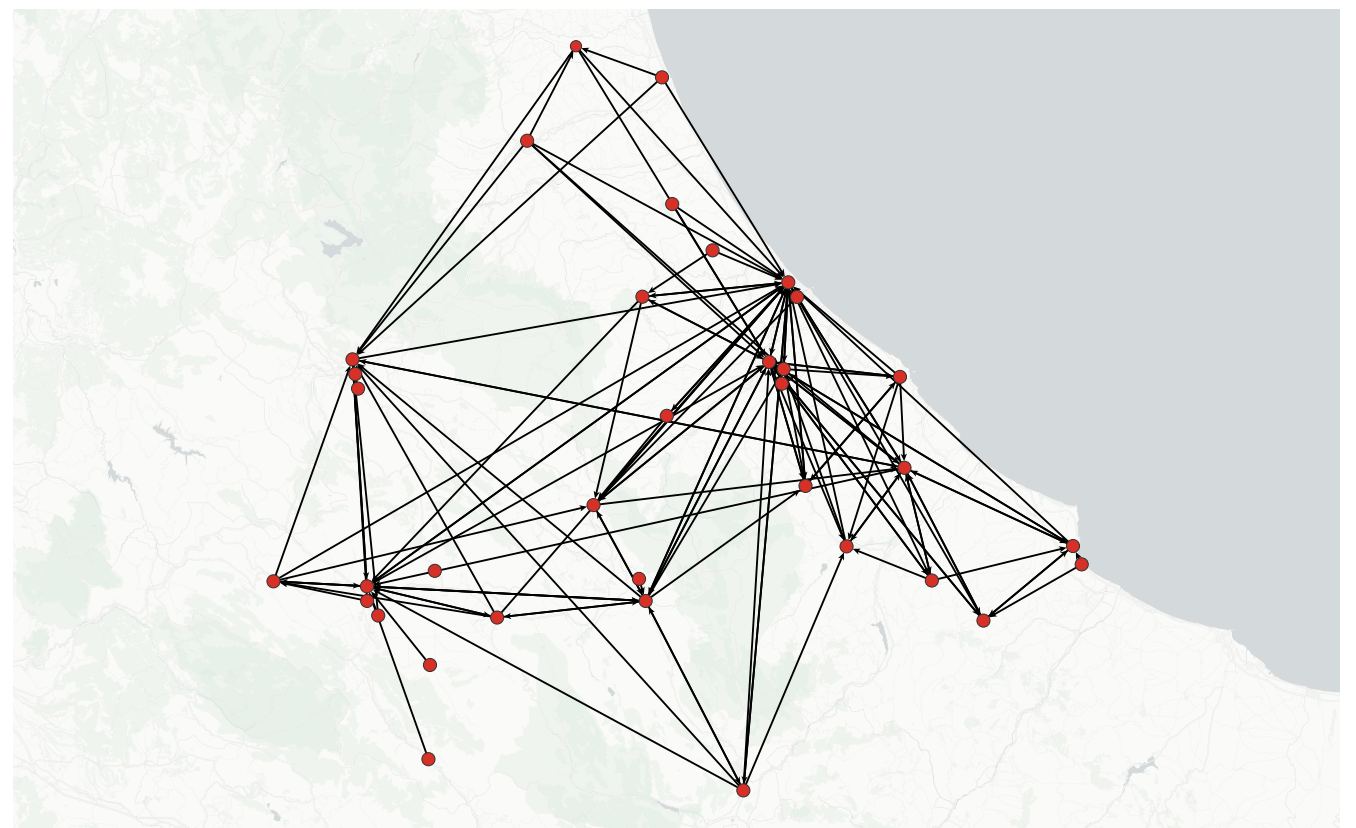

Figure 3: One-mode network for the year 2003. Red circles represent the hospitals and the ties depict the collaborative referral relations among them. The network diagram is superimposed to the geographical map of the region where the hospitals are located. Position of the nodes corresponds to the location of the hospitals.

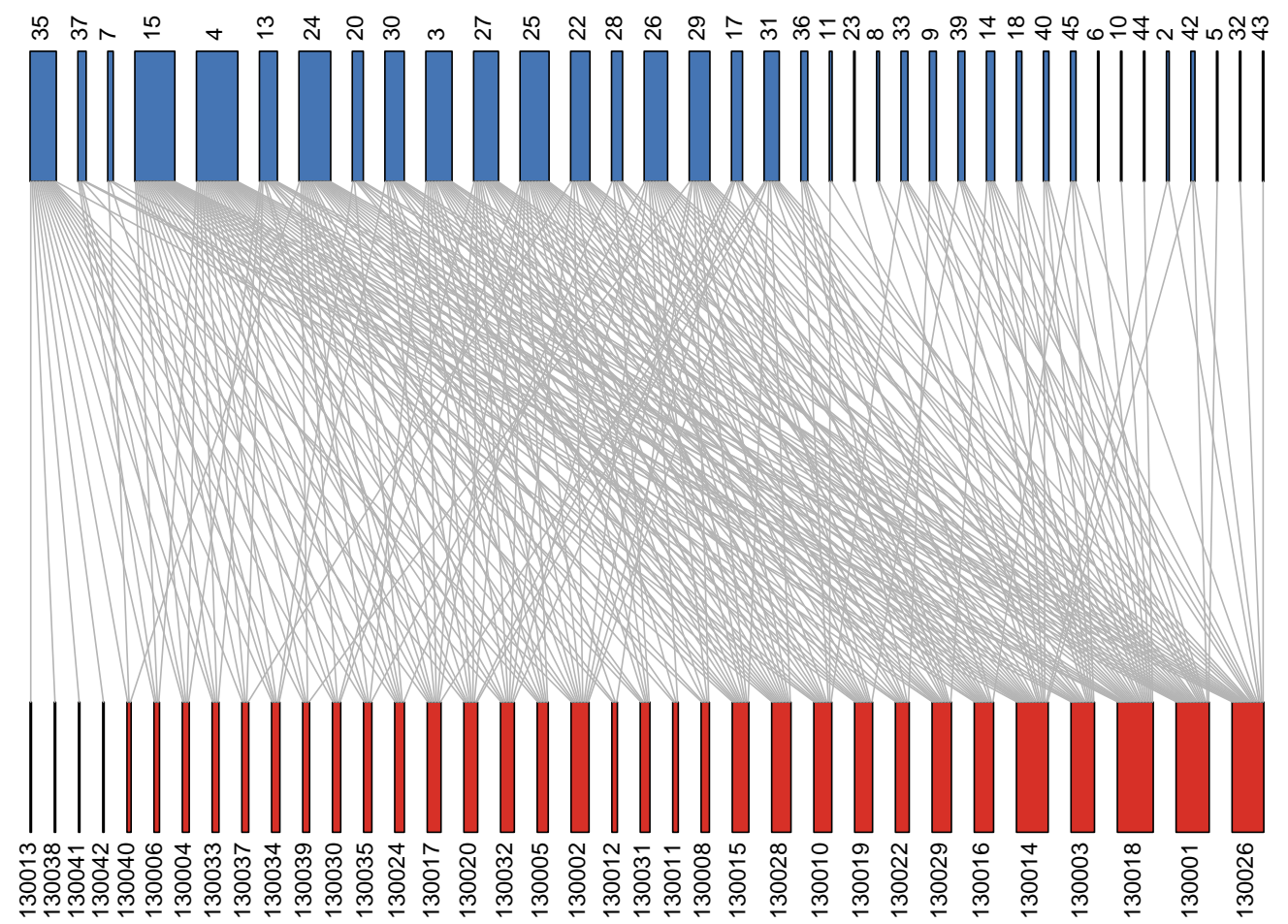

Figure 4: Two-mode network for the year 2003. Red and blue rectangles represent the hospitals and the activities, respectively. Ties indicate which activity belongs to a portfolio of a hospital. The width of a rectangle is proportional to the number of ties incident to that rectangle. 

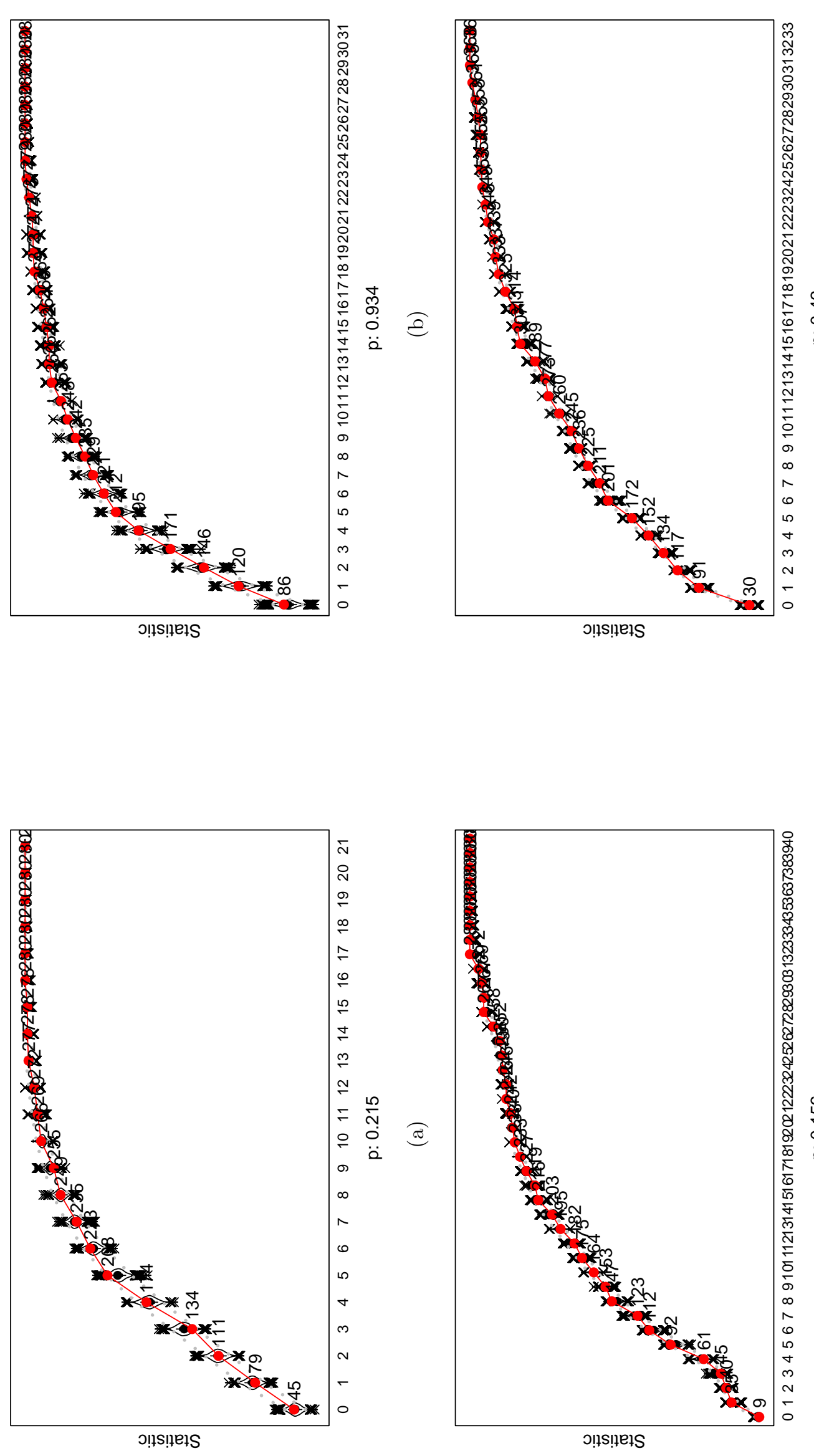